\section{Desigualdad en recursos financieros de la Atención Primaria de Salud Municipal en Chile, 2001-2013}

\author{
CAMILO RIQUELME BRICEÑO ${ }^{1}$, JUAN HAASE DELGADO ${ }^{2, a}$, \\ SEBASTIÁN LAVANDEROS BUNOUT ${ }^{3}$, ANDREA MORALES MARTÍNEZ A $^{4,5, a, b}$
}

\section{Inequality of financial resources for primary health care}

Background: The availability of health care resources is one of the main factors influencing health care inequalities. Aim: To evaluate communal inequality in financial resources for municipal primary care in Chile. Material and Methods: The evolution of income, expenditure and investment per subject enrolled was assessed for the period 2001-2013 in the 320 municipalities that administer municipal health. Inequality was evaluated using Lorenz curves and annual indicators (Gini, Theil, Coefficient of Variation and Reason 90/10). For a panel analysis with fixed effects, the association of these resources with socioeconomic variables and municipal health was evaluated. Results: The Gini of per capita spending increased 1.75 times, while the Gini investment grew by $30 \%$. Per capita spending was significantly and inversely associated with poverty, dependency on the Municipal Common Fund, resources coming from the City Hall, spending per capita, expenses in personnel, performance and investment, rate of years of potential life lost and infant mortality. A direct and significant association was observed with global, less than two years and greater than 64 years urban population, destination of municipal revenue to health, per capita investment in health, rate of healthcare professionals, number of attentions and health care centers and availability of primary emergency facilities. Per capita investment was inversely associated with the number of health care centers and directly to the Fund dependency, municipal health coverage and personnel expenses. Conclusions: Inequality in municipal health funds increased substantially and were associated with social vulnerability, access to municipal health and community health outcomes.

(Rev Med Chile 2017; 145: 723-733)

Key words: Health Disparities; Health Resources; Primary Health Care; Socioeconomic Factors.

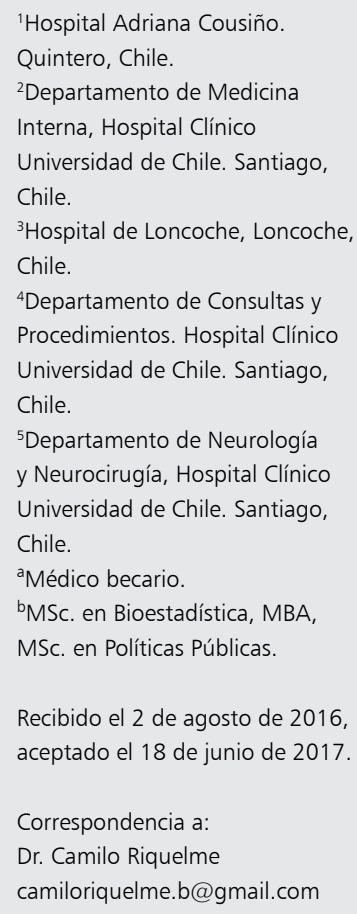

$\mathrm{P}$ ara abordar las desigualdades en salud, la declaración de Alma-Ata de 1978 consideró prioritario potenciar la atención primaria de salud (APS), como pilar fundamental de los sistemas sanitarios ${ }^{1}$. Existe evidencia de que una APS robusta genera mejores resultados sanitarios, mayor equidad y un uso más eficiente de recursos ${ }^{2,3}$. Se ha observado que el impacto de la inversión en APS sobre la equidad, sería incluso mayor que el de la inversión en el sistema de salud general ${ }^{4}$.

Si bien Chile destaca por sus indicadores sanitarios, comparables con países desarrollados, posee la mayor desigualdad de ingresos de la Organización para la Cooperación Económica y el Desarrollo (OECD) y una baja redistribución por beneficios sociales e impuestos ${ }^{5}$; lo que, teó- 
ricamente, podría afectar la distribución de los indicadores sanitarios a lo largo del territorio. A modo de ejemplo, un análisis de panel sobre la tasa de mortalidad infantil (TMI) comunal mostró que, mientras la mediana nacional se mantuvo estable entre 2001 y 2011, su desigualdad aumentó ${ }^{6}$.

La gestión de la APS en Chile está a cargo principalmente de los municipios, contemplando un financiamiento compartido con el gobierno central, del cual recibe transferencias, fundamentalmente, a través de montos capitados (calculados en base a población inscrita), ajustados por factores socioeconómicos y demográficos. Así, la suma de aportes estatales y municipales resulta de proporciones y montos variables en cada comuna y $\mathrm{año}^{7}$. Este sistema de financiamiento mixto con administración municipal podría generar heterogeneidad en las decisiones de inversión y distribución de los gastos comunales en APS, contribuyendo, eventualmente, a la generación de desigualdad de acceso y resultados entre los distintos territorios.

La APS en Chile otorga cobertura, aproximadamente, a $68 \%$ de la población; $91 \%$ inscrita en APS municipal, $8 \%$ en centros dependientes de servicios de salud estatales y $1 \%$ en centros administrados por organizaciones no gubernamentales ${ }^{8}$. A pesar de la relevancia de la APS para el sistema de salud público, varios autores han evidenciado las carencias del sistema en cuanto al financiamiento, el déficit de recursos financieros y de profesionales, la falta de funcionamiento en red con los niveles de mayor complejidad, la escasa resolutividad, la sobrecarga de exigencias en relación a su capacidad en patologías con garantías explícitas de salud (GES), entre otras ${ }^{9-11}$.

Evaluar cómo se distribuyen los recursos financieros en APS resulta crucial para entender y corregir las desigualdades existentes en salud. El objetivo de este trabajo fue evaluar la evolución y distribución de los recursos a nivel comunal y evaluar si las posibles diferencias tuvieran asociación a factores demográficos, socioeconómicos o del sistema de salud municipal.

\section{Materiales y Métodos}

\section{Diseño}

Estudio observacional, longitudinal y retrospectivo sobre los recursos financieros de la APS municipal chilena, desde 2001 a 2013.

\section{Datos y fuentes}

La población anual inscrita en APS, el ingreso, gasto e inversión anuales en salud municipal de las comunas que administran APS, desde el 2001 al 2013, expresados en moneda nacional del último año, se obtuvo del Sistema Nacional de Información Municipal (SINIM) ${ }^{12}$, dependiente de la Subsecretaría de Desarrollo Regional y Administrativo (SUBDERE). Con esta información se construyeron indicadores financieros (ingreso, gasto e inversión) por inscrito, expresados en dólar norteamericano (USD) (tipo de cambio: \$ 500 de moneda nacional, según promedio 2013).

Se entiende como gasto en salud municipal el total de recursos incurridos para la provisión de servicios de APS (que incluye: gasto en personal, en funcionamiento, en capacitación e inversión $)^{12}$, y como inversión en salud municipal, aquellos recursos destinados al financiamiento de obras civiles y equipamiento para nuevos centros, además de sus reposiciones ${ }^{13}$.

Del SINIM se obtuvo, también, las características demográficas y socioeconómicas de la población comunal, derivadas de la Encuesta de Caracterización Socioeconómica Nacional (CASEN), y otras características presupuestarias y del sistema de salud municipal (Tabla 1).

Tabla 1. Definición de variables comunales anuales

\begin{tabular}{|llcc|}
\hline Variables & Definición & $\begin{array}{c}\text { Unidades de } \\
\text { medida }\end{array}$ & $\begin{array}{c}\text { Fuente } \\
\text { original }\end{array}$ \\
\hline Socioeconómicas y demográficas & $\%$ & CASEN \\
Pobreza & Tasa de pobreza comunal & Años & CASEN \\
Escolaridad & Escolaridad promedio de la población comunal & $\%$ & CASEN \\
Población urbana & Porcentaje de población comunal que reside en zonas & urbanas & \\
& & & \\
\hline
\end{tabular}




\begin{tabular}{|c|c|c|c|}
\hline Densidad de población & Densidad poblacional comunal & Habitantes $/ \mathrm{km}^{2}$ & $\mathrm{INE}^{\mathrm{b}}$ \\
\hline Tasa de natalidad & $\begin{array}{l}\text { Nacidos vivos de acuerdo a la comuna de residencia de la } \\
\text { madre, por cada mil habitantes comunales }\end{array}$ & $\begin{array}{l}\text { Nacidos vivos } / 1.000 \\
\text { habitantes }\end{array}$ & DEISc \\
\hline Población femenina & Porcentaje de población comunal femenina & $\%$ & INE \\
\hline Población menor 2 años & Porcentaje de población inscrita en APS menor de 2 años & $\%$ & FONASA $^{d}$ \\
\hline Población mayor 64 años & Porcentaje de población inscrita en APS mayor de 64 años & $\%$ & FONASA \\
\hline \multicolumn{4}{|c|}{ Recursos financieros municipales } \\
\hline $\begin{array}{l}\text { Dependencia del Fondo } \\
\text { Común Municipal (FCM) }\end{array}$ & $\begin{array}{l}\text { Porcentaje de los ingresos comunales que provienen del Fon- } \\
\text { do Común Municipal (FCM): Mecanismo de redistribución } \\
\text { solidaria de los ingresos propios entre los municipios del país }\end{array}$ & $\%$ & SINIMe \\
\hline Aporte municipal a APS & $\begin{array}{l}\text { Proporción del aporte del municipio a APS respecto del } \\
\text { ingreso total percibido en APS }\end{array}$ & $\%$ & SINIM \\
\hline $\begin{array}{l}\text { Ingresos salud por } \\
\text { inscrito }\end{array}$ & $\begin{array}{l}\text { Total de ingresos percibidos por el municipio para APS por } \\
\text { cada inscrito en establecimientos de salud municipal }\end{array}$ & $M \$ /$ por inscrito & SINIM \\
\hline Gasto salud por inscrito & $\begin{array}{l}\text { Total de gastos del sector salud por inscrito en APS (gasto } \\
\text { en personal, funcionamiento, capacitación e inversión) }\end{array}$ & $\mathrm{M} \$ /$ por inscrito & SINIM \\
\hline Gasto en personal & $\begin{array}{l}\text { Proporción del gasto en personal de APS municipal sobre el } \\
\text { total del gasto en salud municipal }\end{array}$ & $\%$ & SINIM \\
\hline Gasto en funcionamiento & $\begin{array}{l}\text { Proporción del gasto en funcionamiento sobre el total del } \\
\text { gasto en salud municipal }\end{array}$ & $\%$ & SINIM \\
\hline Gasto en capacitación & $\begin{array}{l}\text { Proporción del gasto en capacitación del personal de APS } \\
\text { municipal sobre el total del gasto en APS municipal }\end{array}$ & $\%$ & SINIM \\
\hline Inversión & $\begin{array}{l}\text { Proporción de Inversión Real en APS respecto del total de } \\
\text { gasto en Salud (destinado a infraestructura y equipamiento) }\end{array}$ & $\%$ & SINIM \\
\hline Inversión real por inscrito & $\begin{array}{l}\text { Monto total destinado a inversión en sector salud (destinado } \\
\text { a infraestructura y equipamiento) por inscrito en APS }\end{array}$ & $\mathrm{M} \$ /$ por inscrito & SINIM \\
\hline \multicolumn{4}{|c|}{ Demanda y Oferta de servicios de salud primaria municipal } \\
\hline Cobertura APS & $\begin{array}{l}\text { Población inscrita en el sistema de salud municipal sobre el } \\
\text { total de población comunal }\end{array}$ & $\%$ & $\begin{array}{l}\text { FONASA, } \\
\text { INE }\end{array}$ \\
\hline Tasa de médicos & $\begin{array}{l}\text { Médicos de APS municipal por cada diez mil inscritos en } \\
\text { APS municipal }\end{array}$ & $\begin{array}{l}\text { Médicos } / 10.000 \\
\quad \text { inscritos }\end{array}$ & $\begin{array}{l}\text { SINIM, } \\
\text { FONASA }\end{array}$ \\
\hline $\begin{array}{l}\text { Tasa de otros } \\
\text { profesionales }\end{array}$ & $\begin{array}{l}\text { Profesionales no médicos de APS municipal de por cada } \\
\text { diez mil inscritos en APS municipal }\end{array}$ & $\begin{array}{l}\text { Otros profesionales/ } \\
10.000 \text { inscritos }\end{array}$ & $\begin{array}{l}\text { SINIM, } \\
\text { FONASA }\end{array}$ \\
\hline Tasa de centros & $\begin{array}{l}\text { Centros de APS municipal por cada diez mil inscritos en } \\
\text { APS municipal }\end{array}$ & $\begin{array}{l}\text { Centros/10.000 } \\
\text { inscritos }\end{array}$ & $\begin{array}{l}\text { SINIM, } \\
\text { FONASA }\end{array}$ \\
\hline Tasa de consultas & Consultas médicas por cada mil inscritos en APS municipal & $\begin{array}{l}\text { Consultas } / 1.000 \\
\text { inscritos }\end{array}$ & $\begin{array}{l}\text { SINIM, } \\
\text { FONASA }\end{array}$ \\
\hline Disponibilidad de SAPU & $\begin{array}{l}\text { Disponibilidad de servicios de atención primaria de urgencia } \\
\text { (SAPU) }\end{array}$ & $\begin{array}{l}\text { 0: No disponible } \\
\text { 1: Disponible }\end{array}$ & SINIM \\
\hline Distancia al Hospital Base & $\begin{array}{l}\text { Distancia de la comuna al hospital de referencia, base o } \\
\text { emergencia }\end{array}$ & km. & DEIS, INE \\
\hline \multicolumn{4}{|c|}{ Resultados en Salud comunal } \\
\hline Tasa mortalidad infantil & $\begin{array}{l}\text { Muertes de menores de } 1 \text { año, según la comuna de resi- } \\
\text { dencia de la madre, por cada mil nacidos vivos }\end{array}$ & $\begin{array}{l}\text { Muertes de }<1 \\
\text { Año/1.000 nacidos vivos }\end{array}$ & DEIS \\
\hline Tasa de AVPP & $\begin{array}{l}\text { Años de vida potencialmente perdidos (AVPP) por mil ha- } \\
\text { bitantes de la comuna }\end{array}$ & $\begin{array}{l}\text { AVPP/1.000 } \\
\text { habitantes }\end{array}$ & DEIS \\
\hline
\end{tabular}

aCASEN: Encuesta de Caracterización Socioeconómica Nacional; ' ${ }^{a}$ NE: Instituto Nacional de Estadísticas; ' $D E I S:$ Departamento de Estadísticas e Información de Salud; ' $F$ FONASA: Fondo Nacional de Salud; eSINIM: Sistema Nacional de Información Municipal. 


\section{Análisis estadístico}

i) Evolución de los recursos financieros: Se evaluó gráficamente la evolución de cada indicador a nivel comunal en el período.

ii) Análisis de desigualdad: Se construyeron curvas de Lorenz para graficar la evolución de la desigualdad de cada indicador. El eje horizontal representa el porcentaje acumulado de comunas y el vertical, el indicador acumulado que recibe cada porcentaje acumulado de comunas. En perfecta igualdad (recta de $45^{\circ}$ ), cada porcentaje acumulado de comunas recibe o destina igual acumulado de ingreso, gasto o inversión; en tanto, en completa desigualdad, una comuna abarcaría todos los recursos del país ${ }^{14}$.

Para cuantificar la distribución se construyeron índices anuales de desigualdad (índices de Gini, Theil, coeficiente de variación y razón 90/10).

iii) Análisis de asociación: Las asociaciones entre variables comunales y gasto o inversión en salud municipal por inscrito fueron evaluadas por regresión con datos de panel con los 13 años de estudio. Esta metodología provee información sobre el comportamiento individual, a través del tiempo y a través de los individuos. Su principal ventaja sobre la regresión clásica radica en la precisión de las estimaciones al incrementar el número de observaciones, combinando varios períodos de tiempo para cada unidad de análisis ${ }^{15}$.

La estimación de coeficientes se realizó a partir del modelo de efectos fijos, previa validación por test de Hausman y corrección por heterocedasticidad, de acuerdo a la expresión:

$$
\text { Gasto/Inversión per cápita }{ }_{i t}=\alpha_{i}+\beta \chi_{i t}^{\prime}+\varepsilon_{i t}
$$

donde, $i$ representa las comunas y $t$ los años evaluados, $\alpha_{i}$ representa el efecto individual (comunal), $\beta$ representa los coeficientes de asociación, $\chi_{i t}^{\prime}$ corresponde a la matriz de variables explicativas para cada comuna y año y $\varepsilon_{i t}$ es el término de error. Así, el modelo de efectos fijos permite corregir posibles sesgos por omisión de variables relevantes no observables heterogéneas entre comunas y que pudieran estar relacionadas con otras variables explicativas ${ }^{16}$.

La base de datos y su análisis se realizó con STATA ${ }^{\circledR} 12.0$, a partir de estadísticas comunales disponibles en los sitios web de las instituciones mencionadas, por lo cual no se requirió aprobación previa por un comité de ética.

\section{Resultados}

\section{Evolución de los recursos financieros en salud municipal}

Las medianas de ingreso y gasto en APS por inscrito en el período fueron de USD 99,5 (RI: 68,9; rango: 0-22.588) y USD 98,1 (RI: 67,5; rango: 0-23.211), respectivamente. Ambas cifras presentaron tendencias crecientes en el período, con aumentos en torno a 1,6 veces: mientras la mediana del ingreso anual aumentó de USD 62,9 en 2001 a USD 163,6 en 2013; la del gasto aumentó de USD 63,0 a USD 166,5.

En tanto, la inversión tuvo una mediana de cero en el período (RI: 0,6; rango: USD 0-128); sin embargo, su evolución fluctuó. Mientras que el p25 se mantuvo en cero, la mediana y el p75 presentaron un aumento sostenido entre los años 2004 y 2006, llegando a USD 0,5 y USD 2,5 anuales por inscrito, respectivamente, con bruscas caídas posteriores, manteniéndose en cero desde el año 2008 (Figura 1).

\section{Desigualdad en recursos financieros en salud municipal}

Las curvas de Lorenz mostraron un progresivo aumento de la desigualdad entre los años 2001, 2007 y 2013, en los tres indicadores financieros; observándose la mayor brecha en la inversión por inscrito, con un aumento más acentuado entre 2007 y 2013 (Figura 2).

Los índices de desigualdad en ingresos y gastos por inscrito tendieron al aumento, con un abrupto ascenso en 2010, con recuperación gradual. En particular, el índice de Gini de ingresos por inscrito fluctuó de 0,17 en 2001 a 0,46 en $2013(0,68$ el 2010); en tanto, el Gini de gastos varió de 0,16 a 0,44 en el período $(0,68$ el 2010). Respecto a la inversión, hubo un incremento similar, pero de magnitudes sustantivamente mayores: el índice de Gini varió de 0,73 a 0,95 en el período $(0,96$ en 2008 y 0,97 en 2011) (Figura 3 ).

\section{Factores asociados a la disponibilidad de recursos financieros}

El panel de gasto por inscrito en APS reveló una asociación inversa al porcentaje de pobreza comunal $(\mathrm{p}<0,01)$ y directa al porcentaje de 

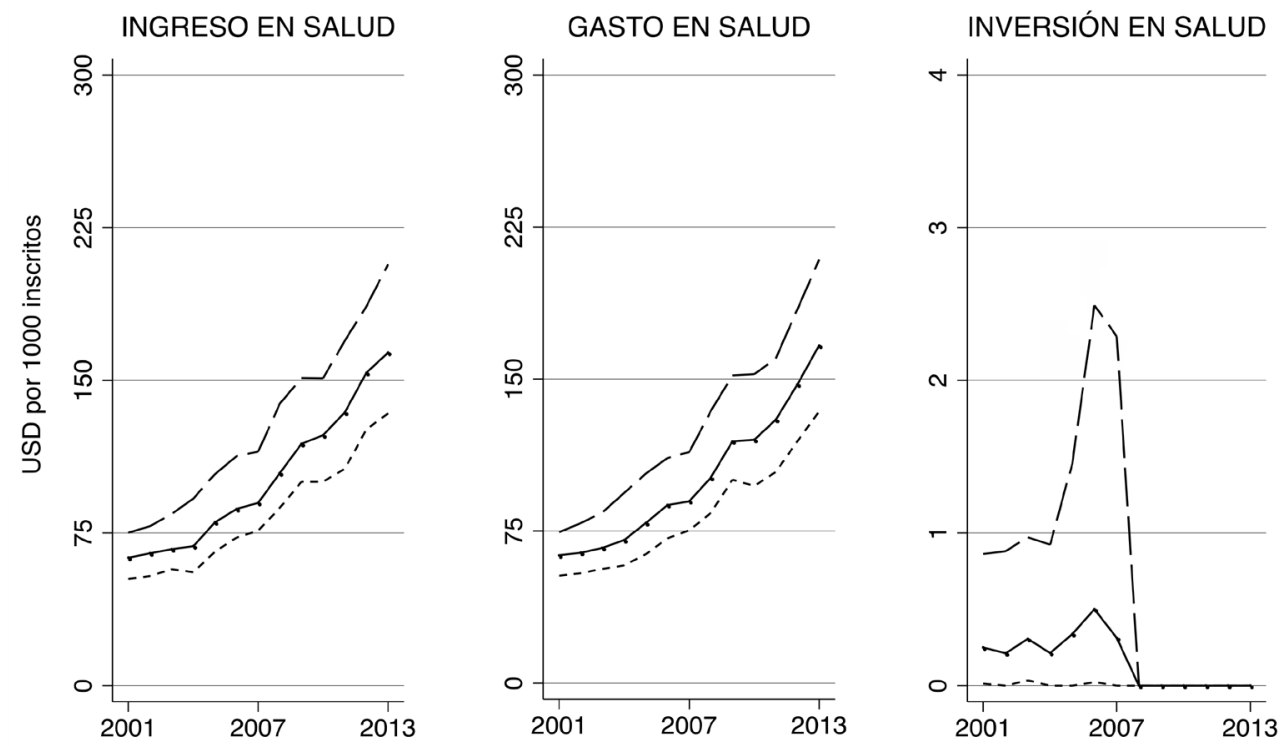

$\because \mathrm{p} 50 \quad \ldots$ p25 - $\mathrm{p} 75$

Figura 1. Evolución del ingreso, gasto e inversión en salud municipal por inscrito. Chile, 2001-2013.
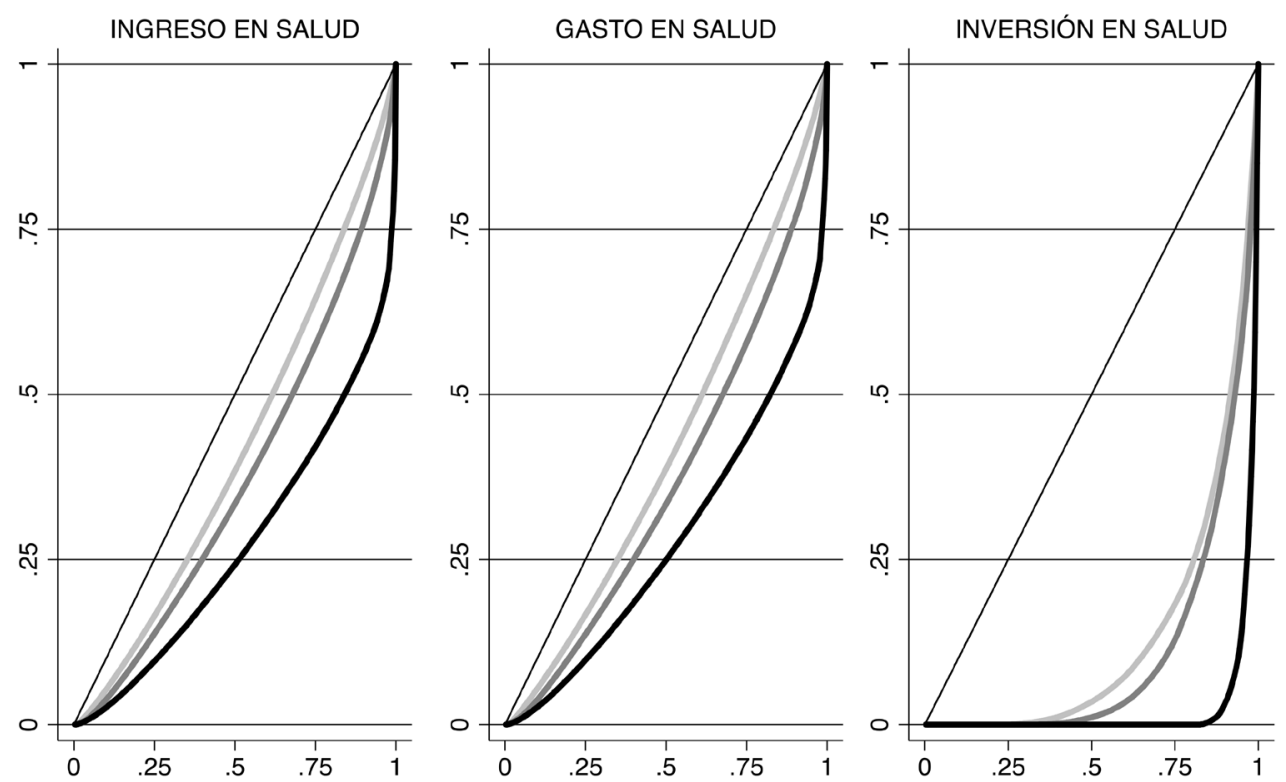

$-2001-2007-2013$

Figura 2. Curvas de Lorenz: ingreso, gasto e inversión en salud municipal por inscrito. Chile, 2001-2013. 


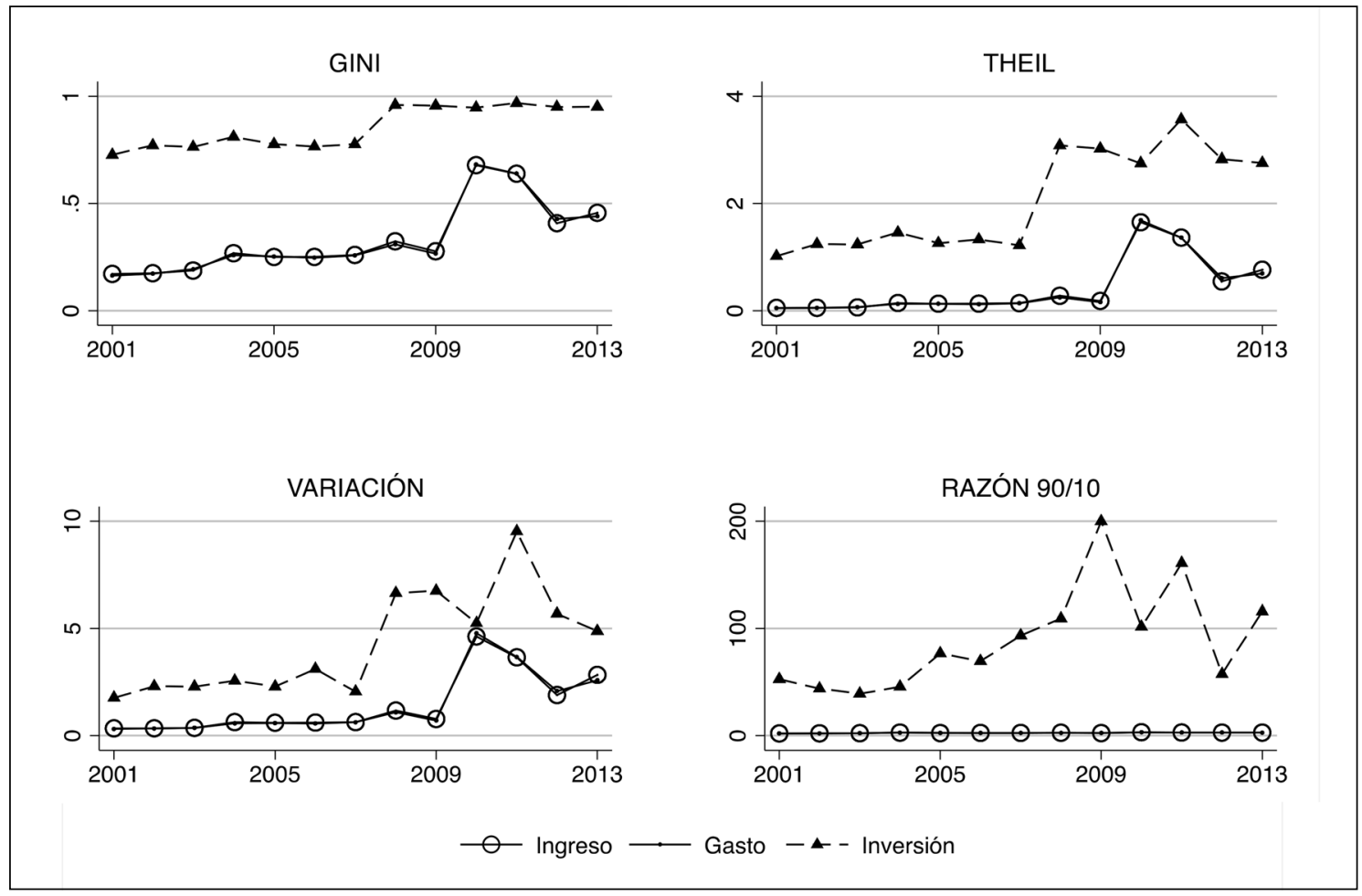

Figura 3. Evolución de la desigualdad de ingresos, gastos e inversión en salud municipal per cápita. Chile, 2001-2013.

población urbana $(\mathrm{p}<0,01)$, menores de 2 años ( $\mathrm{p}<0,01$ o 0,05 , según el modelo) y mayores de $64(\mathrm{p}<0,01)$. En tanto, la asociación a escolaridad resultó directa, pero significativa, sólo en modelos que no incluyeron la tasa de años de vida potencialmente perdidos por muertes prematuras (AVPP) (Tabla 2, A y C). Para testear este efecto, se evaluó la relación entre escolaridad y tasa de AVPP, encontrando una alta correlación inversa (rho $=-0,4086, p<0,001)$ y una asociación que refleja que por cada 1 año más de escolaridad la tasa de AVPP disminuye en 22,2 años por 1.000 habitantes $(\mathrm{p}<0,001)$; lo cual explicaría lo ocurrido en los modelos de panel.

En relación al financiamiento y la oferta de servicios de APS, se observaron asociaciones inversas del gasto por inscrito a dependencia del Fondo Común Municipal (FCM) ( $\mathrm{p}<0,01$ o 0,05, según el modelo), proporción del ingreso total de APS que proviene del aporte municipal ( $\mathrm{p}<0,01)$, proporción del gasto en APS destinado al pago del personal $(\mathrm{p}<0,05)$, al funcionamiento $(\mathrm{p}<0,1) \mathrm{y}$ a la inversión $(\mathrm{p}<0,01)$. En tanto, se encontraron asociaciones directas a la proporción de ingresos municipales que son destinados a APS $(\mathrm{p}<0,01)$, inversión en salud por inscrito $(\mathrm{p}<0,01)$, tasa de profesionales sanitarios no médicos $(\mathrm{p}<0,01)$, de centros de APS $(\mathrm{p}<0,01)$ y de consultas médicas $(\mathrm{p}<0,01)$ y disponibilidad de SAPU en la comuna $(\mathrm{p}<0,05 \mathrm{o}<0,1$, según el modelo). En el caso de la tasa de centros por inscrito destaca que por cada punto de aumento el gasto por inscrito aumenta más de 100 veces (Tabla 2, A-D).

$\mathrm{Al}$ incluir indicadores de resultados en salud, se observó que el gasto por inscrito se asoció directa y significativamente, tanto a la tasa de AVPP ( $\mathrm{p}<0,01)$, como a la TMI comunal $(\mathrm{p}<0,05)$ (Tabla 2, B-D).

En tanto, el panel de inversión per cápita mostró asociación inversa a la tasa de centros $(\mathrm{p}<0,05)$ y asociación directa a dependencia del FCM $(\mathrm{p}<0,05 \mathrm{o}<0,1)$, gasto en personal $(\mathrm{p}<0,05)$ y cobertura de APS comunal $(\mathrm{p}<0,01)$ (Tabla 3). 
Tabla 2. Estimación de gasto por inscrito en APS municipal. Chile, 2001-2013

\begin{tabular}{|c|c|c|c|c|}
\hline Variables & (2A) & (2B) & $(2 \mathrm{C})$ & (2D) \\
\hline Pobreza & $-1,5408^{\star \star \star}$ & $-1,0908^{* * *}$ & $-1,5120^{* * *}$ & $-1,0443^{* * *}$ \\
\hline Escolaridad & $0,0431^{\star *}$ & 0,0132 & $0,0440^{* *}$ & 0,0127 \\
\hline Población urbana & $1,2613^{* * *}$ & $1,2611^{\star * *}$ & $1,1842^{* * \star}$ & $1,1899^{* * *}$ \\
\hline Densidad de población & $-0,0038$ & $-0,0053$ & $-0,0039$ & $-0,0056$ \\
\hline Tasa de natalidad & $-0,9204^{*}$ & $-0,6144$ & $-0,8979$ & $-0,5737$ \\
\hline Población femenina & 1,6993 & 2,4058 & 2,7830 & 3,7329 \\
\hline Población menor 2 años & $2,2765^{\star \star}$ & $3,8073^{* * *}$ & $2,3647^{* *}$ & $3,9968^{* * *}$ \\
\hline Población mayor 64 años & $12,4329^{* * *}$ & $10,5404^{* * *}$ & $12,8343^{* * *}$ & $10,9147^{* * *}$ \\
\hline Dependencia FCM & $-0,9186^{* * *}$ & $-0,6374^{* * *}$ & $-0,9674^{* * *}$ & $-0,6696^{* * *}$ \\
\hline Aporte municipal a APS & $-0,7543^{* \star *}$ & $-0,7152^{\star \star \star}$ & $-0,7290^{* * *}$ & $-0,6944^{* * *}$ \\
\hline Ingresos municipales a APS & $3,8008^{* * *}$ & $3,2099^{* * *}$ & $3,7041^{* * *}$ & $3,1310^{* * *}$ \\
\hline Gasto en personal & $-1,7348^{\star \star}$ & $-1,4734^{\star *}$ & $-1,6749^{* *}$ & $-1,4213^{* *}$ \\
\hline Gasto en funcionamiento & $-1,3252^{*}$ & $-1,2864^{*}$ & $-1,2561^{*}$ & $-1,2340^{*}$ \\
\hline Gasto en capacitación & 2,3435 & 1,4024 & 2,5343 & 1,6081 \\
\hline Inversión & $-3,6552^{\star \star \star}$ & $-3,2624^{\star * *}$ & $-3,5813^{* * *}$ & $-3,1947^{* * *}$ \\
\hline Inversión real por inscrito & $1,9278^{* * *}$ & $1,9072^{* * *}$ & $1,9019^{* * *}$ & $1,8819^{\star * \star}$ \\
\hline Cobertura APS & 0,1268 & 0,1089 & 0,1308 & 0,1081 \\
\hline Tasa de médicos & $-0,1026$ & $-0,1162$ & $-0,0888$ & $-0,1112$ \\
\hline Tasa otros profesionales & $0,1626^{* * *}$ & $0,1408^{* * *}$ & $0,1498^{* * *}$ & $0,1316^{* * *}$ \\
\hline Tasa de centros & $103,8038^{* * *}$ & $105,6530^{* * *}$ & $104,1609^{* * *}$ & $105,9588^{* \star \star}$ \\
\hline Tasa de consultas & $0,3376^{* \star *}$ & $0,2255^{* * *}$ & $0,3661^{* * *}$ & $0,2466^{* * *}$ \\
\hline SAPU & $5,8620^{*}$ & $6,3506^{* *}$ & $6,0926^{* *}$ & $6,7468^{* *}$ \\
\hline Distancia Hospital Base & 0,0067 & $-0,0040$ & 0,0033 & $-0,0064$ \\
\hline Tasa AVPP & & $-0,0024^{* * *}$ & & $-0,0024^{* * *}$ \\
\hline Tasa mortalidad infantil & & & $-0,3226^{* *}$ & $-0,2247^{\star *}$ \\
\hline Constante & $-6,1820$ & $-53,9632$ & $-61,7660$ & $-123,1305$ \\
\hline $\mathrm{R}^{2}$ within & 0,8244 & 0,8710 & 0,8271 & 0,8739 \\
\hline Observaciones & 2.747 & 2.527 & 2.678 & 2.458 \\
\hline
\end{tabular}


Tabla 3. Estimación de inversión real por inscrito en APS municipal. Chile, 2001-2013

\begin{tabular}{|c|c|c|c|c|}
\hline Variables & (3A) & (3B) & (3C) & (3D) \\
\hline Pobreza & 0,0120 & 0,0148 & 0,0129 & 0,0159 \\
\hline Escolaridad & $-0,0007$ & $-0,0002$ & $-0,0007$ & $-0,0002$ \\
\hline Población urbana & $-0,0104$ & $-0,0128$ & $-0,0131$ & $-0,0152$ \\
\hline Densidad de población & $-0,0000$ & $-0,0001$ & $-0,0000$ & $-0,0001$ \\
\hline Tasa de natalidad & 0,0235 & 0,0240 & 0,0235 & 0,0232 \\
\hline Población femenina & 0,0615 & 0,0522 & 0,0489 & 0,0296 \\
\hline Población menor 2 años & 0,0333 & 0,0064 & 0,0337 & 0,0063 \\
\hline Población mayor 64 años & 0,0434 & 0,0284 & 0,0514 & 0,0348 \\
\hline Dependencia FCM & $0,0207^{*}$ & $0,0275^{\star *}$ & $0,0215^{\star}$ & $0,0289^{* *}$ \\
\hline Aporte municipal a APS & $-0,0099$ & $-0,0123$ & $-0,0100$ & $-0,0126$ \\
\hline Ingresos municipales a APS & $-0,0102$ & $-0,0022$ & $-0,0112$ & $-0,0030$ \\
\hline Gasto salud por inscrito & $-0,0304$ & $-0,0468$ & $-0,0325$ & $-0,0493$ \\
\hline Gasto en personal & $0,0115^{\star *}$ & $0,0162^{\star *}$ & $0,0116^{\star *}$ & $0,0164^{* *}$ \\
\hline Gasto en funcionamiento & $-0,0039$ & $-0,0003$ & $-0,0035$ & 0,0002 \\
\hline Gasto en capacitación & $-0,0179$ & $-0,0131$ & $-0,0186$ & $-0,0139$ \\
\hline Inversión & $0,2366^{*}$ & 0,1951 & 0,2363 & 0,1925 \\
\hline Cobertura APS & $1,1541^{\star \star \star}$ & $1,1402^{* * *}$ & $1,1609^{* * *}$ & $1,1475^{* * *}$ \\
\hline Tasa de médicos & 0,0042 & 0,0048 & 0,0047 & 0,0052 \\
\hline Tasa de otros profesionales & $-0,0017$ & $-0,0022$ & $-0,0018$ & $-0,0023$ \\
\hline Tasa de centros de APS & $-1,0507^{* *}$ & $-1,5790^{* *}$ & $-1,0545^{* *}$ & $-1,5960^{* *}$ \\
\hline Tasa de consultas & $-0,0019$ & $-0,0033$ & $-0,0022$ & $-0,0038$ \\
\hline SAPU & $-0,2378$ & $-0,1414$ & $-0,2458$ & $-0,1472$ \\
\hline Distancia Hospital Base & $-0,0012$ & $-0,0009$ & $-0,0010$ & $-0,0007$ \\
\hline Tasa AVPP & & 0,0000 & & 0,0000 \\
\hline Tasa mortalidad infantil & & & $-0,0028$ & $-0,0059$ \\
\hline Constante & $-39,411$ & $-43,967$ & $-32,636$ & $-32,428$ \\
\hline $\mathrm{R}^{2}$ within & 0,6561 & 0,6502 & 0,6569 & 0,6511 \\
\hline Observaciones & 2.747 & 2.527 & 2.678 & 2.458 \\
\hline
\end{tabular}




\section{Discusión}

Estos resultados reflejan una desigualdad creciente en recursos financieros entre las comunas que administran APS, lo que se evidencia en una amplia dispersión de la disponibilidad presupuestaria por inscrito entre las distintas comunas del país, la cual no necesariamente se condice con las necesidades poblacionales existentes.

Al cuantificar la desigualdad, los indicadores mostraron tener similares comportamientos en el período. Sin embargo, dos años destacan en relación a las fluctuaciones observadas: i) la desigualdad en inversión presentó un alza brusca en 2008, que podría estar asociada a la crisis subprime que afectó la economía mundial; ii) todos los indicadores tienden a una abrupta elevación en 2010, que podría relacionarse al terremoto que afectó a buena parte del país a principios de ese año y que significó una redistribución del presupuesto nacional.

$\mathrm{Al}$ evaluar los factores sociodemográficos determinantes del gasto se encontraron asociaciones relevantes: i) El aumento del gasto por inscrito a mayor proporción de población en edades extremas se condice con la evidencia existente sobre el mayor uso de servicios sanitarios ${ }^{17,18}$; ii) Resulta destacable la fuerte asociación inversa entre escolaridad y AVPP, esto se relaciona con los hallazgos de la literatura que han mostrado asociación entre el nivel socioeconómico y AVPP ${ }^{19-21}$; iii) El análisis de panel mostró que, a mayor proporción de población comunal en condiciones de pobreza y ruralidad, reconocidos determinantes sociales ${ }^{22-25}$, menor fue el gasto por inscrito, a pesar de que el sistema de asignación per cápita del gobierno central considera ajustes por ambos factores ${ }^{7}$. Esto revela un escenario de franca desigualdad socioeconómica y territorial, donde habitantes de comunas con perfiles vulnerables tienen menor posibilidad de acceder a atención sanitaria.

Arteaga et al, basados en datos de la CASEN de 1998, reportaron una correlación directa entre ingresos domésticos medios y el aporte municipal por beneficiario, con una compensación parcial del aporte del gobierno central hacia comunas más pobres $^{26}$. Nuestros hallazgos revelarían que en el período, esta asociación no sólo no ha logrado ser corregida, sino que más bien se ha profundizado la desigualdad estructural que perpetúa la desigualdad de oportunidades básicas en salud.
Por otra parte, se observa que diversas variables de oferta de salud municipal constituyen determinantes centrales del gasto por inscrito: mayor tasa de profesionales sanitarios, disponibilidad de centros, tasa de consultas médicas y contar con servicios de urgencia primaria. Igualmente relevante resultó la capacidad financiera de los municipios para sostener esta oferta: las comunas con mayor gasto por inscrito muestran menor dependencia del fondo redistributivo de ingresos, mayor proporción de sus ingresos a APS, con mayor inversión en salud por inscrito y cuyo gasto no requiere destinarse prioritamente al pago del personal o del funcionamiento de sus centros.

Comentario especial merece el efecto de la tasa de centros cuyo aumento en 1 punto aumenta en más de 100 veces el gasto por inscrito comunal. Esto revela la complejidad de la expansión de la oferta de servicios de APS, a través de la construcción de nuevos centros que permitan disminuir la densidad de inscritos, si se considera que el sistema de financiamiento de APS coloca en desventaja a aquellas comunas que no cuentan con una capacidad de generación de recursos propios para la destinación y mantención de servicios de salud municipal.

En resumen, comunas que tienen más posibilidades de resolver sus necesidades sanitarias locales generan mayor gasto por inscrito en APS, planteando la inquietud de que las comunas gasten de acuerdo a su capacidad financiera y no en función de las características y necesidades de su población. Esta discordancia probablemente se origina por la disparidad entre la determinación de objetivos sanitarios para la APS desde el gobierno central, su administración por el gobierno local y un financiamiento compartido entre ambos.

Asimismo, destacan las asociaciones encontradas a indicadores de resultados sanitarios: comunas con mayor gasto por inscrito en APS presentan menores tasas de mortalidad infantil y de años de vida perdidos por muertes prematuras. Estos resultados concuerdan con la evidencia presentada por Morales et al. ${ }^{6}$, la cual asoció significativamente la mortalidad infantil comunal al gasto en salud municipal per cápita, y con la evidencia de Sánchez et al, que mostró 37\% más de muertes prematuras en adultos del quintil más pobre, comparado con el más rico ${ }^{21}$.

El panel de inversión por inscrito reveló que ésta crece a mayor dependencia del FCM, cober- 
tura de APS, proporción de gasto en personal y menor tasa de centros. Considerando que las comunas con mayor gasto por inscrito presentaban, proporcionalmente, menor destinación del gasto a inversión, las asociaciones reveladas en este último panel muestran que las políticas de inversión del gobierno central (destinadas al financiamiento de obras y equipamiento para nuevos centros) han sido enfocadas apropiadamente en zonas con mayor demanda y menor oferta de servicios. Sin embargo, se esperaría que otros determinantes de la demanda, como pobreza, ruralidad, distancia al hospital base o mortalidad infantil comunal, hubiesen resultado igualmente asociados, evidenciando que las políticas de inversión en APS en Chile aún no se encuentran completamente enfocadas hacia la oportunidad de acceso a servicios y resultados en salud, lo que ahondaría la inequidad existente en el sistema.

Entendiendo inequidad en salud como lo define Whitehead: "diferencias innecesarias y evitables pero que, además, se consideran arbitrarias e injustas"27, se comprende la necesidad de encauzar esfuerzos para revertir estas asociaciones y evitar que la desigualdad perpetúe.

\section{Referencias}

1. Organización Mundial de la Salud. Declaración de Alma-Ata. Conferencia Internacional sobre Atención Primaria de Salud, Alma-Ata, URSS, 6-12 de septiembre de 1978.

2. Starfield B, Shi L, Macinko J. Contribution of primary care to health systems and health. Milbank Q 2005; 83: 457-502.

3. Kringos DS, Boerma WG, Hutchinson A, van der Zee J, Groenewegen PP. The breadth of primary care: a systematic literature review of its core dimensions. BMC Health Serv Res 2010; 10: 65.

4. Starfield B. State of the Art in Research on Equity in Health. J Health Polit Policy Law 2006, 31: 11-32.

5. OECD, Society at a Glance 2014, Highlights: Chile-OECD Social Indicators; 2004.

6. Morales A, Landerretche O, Martínez A. Mortalidad infantil en Chile: Un indicador de desigualdad. Trabajos de Investigación en Políticas Públicas 2015; 20: 2-1.

7. Raña J, Ferrer JC, Bedregal P. Modelo de asignación de recursos en atención primaria. Rev Med Chile 2007; 135: 54-62.

8. Ministerio de Salud, Gobierno de Chile. Departamento de Estadísticas e Información en salud. Población Beneficiaria de FONASA 2012. Disponible en: http://www. deis.cl/estadisticas-poblacion/

9. Montero J, Tellez A, Herrera C. Reforma sanitaria chilena y la atención primaria de salud. Algunos aspectos críticos. Centro de Políticas Públicas UC 2010; 5 (38): 3-12.

10. Arancibia E, Contreras JC, Fábrega R, Infante A, Irarrázabal I, Inostoza M, et al. Fortalecimiento de la atención primaria de salud: propuestas para mejorar el sistema sanitario chileno. Centro de políticas públicas UC 2014; 76: 6-20.

11. Bass C. Modelo de salud familiar en Chile y mayor resolutividad de la atención primaria de salud: ¿contradictorios o complementarios? Medwave 2012; 12 (11): e5571.

12. Ministerio del Interior, Gobierno de Chile. Subsecretaría de Desarrollo Regional y Administrativo (SUBDERE). Sistema Nacional de Información Municipal (SINIM). Disponible en: http://www.sinim.gov.cl/

13. Ministerio de Hacienda. Dirección de Presupuestos. Sistema público de salud. Situación actual y Proyecciones fiscales 2013-2050. Diciembre, 2013.

14. Deaton A. The Analysis of Household Surveys. A Microeconometric Approach to Development Policy. Washington, DC: The World Bank 1997; 133-35.

15. Labra R, Torrecillas C. Guía CERO para datos de panel. Un enfoque práctico. (Working Paper \# 2014/16). UAM-Accenture Working Papers. (Internet) Madrid, 2014 (consultado el 26 de enero de 2016). Disponible en: https://www.uam.es/docencia/degin/catedra/documentos/16_Guia\%20CERO\%20para\%20datos\%20 de\%20panel_Un\%20enfoque\%20practico.pdf

16. Contreras D. Pobreza, desigualdad, bienestar y políticas sociales. Elementos metodológicos para el debate. Serie Docente N. 16. Santiago: Corporación de Investigaciones Económicas para América Latina, 1998; 37-9.

17. Vargas V, Wasem J. Risk Adjustment and Primary Health Care in Chile. Croat Med J 2006; 47 (3): 459-68.

18. van de Ven WP, Ellis RP. Risk adjustment in competitive health plan markets. In: Culyer AJ, Newhouse JP, editors. Handbook of health economics. Amsterdam: Elsevier Science B.V; 2000. p. 755-845.

19. Gattini C, Sanderson C, Castillo-Salgado C. Variación de los indicadores de mortalidad evitable entre comunas chilenas como aproximación a las desigualdades de salud. Rev Panam Salud Publica 2002; 12 (6): 454-61.

20. GBD 2015 DALYs and HALE Collaborators. Global, regional, and national disability-adjusted life-years (DALYs) for 315 diseases and injuries and healthy life expectancy (HALE), 1990-2015: a systematic analysis for 
the Global Burden of Disease Study 2015. Lancet 2016; 388: 1603-58.

21. Sánchez H, Albala C, Lera L. Años de vida perdidos por muerte prematura (AVPP) en adultos del Gran Santiago: ¿Hemos ganado con equidad?. Rev Med Chile 2005; 133 (5): 575-82.

22. Smith KB, Humphreys JS, Wilson MG. Addressing the health disadvantage of rural populations: how does epidemiological evidence inform rural health policies and research? Aust J Rural Health 2008; 16: 56-6.

23. Wagstaff A. Pobreza y desigualdades en el sector de la salud. Rev Panam Salud Publica 2002; 11 (5-6): 316-26. 24. Bedregal P, Zavala C, Atria J, Núñez G, Pinto M, Valdés $\mathrm{S}$. Acceso a redes sociales y de salud de población en extrema pobreza. Rev Med Chile 2009; 137 (6): 753-8.

25. Olavarría M. Acceso a la salud en Chile. Acta Bioethica 2005; año XI, 1: 47-64.

26. Arteaga O, Thollaug S, Nogueira A, Darras C. Información para la equidad en salud en Chile. Rev Panam Salud Publica 2002; 11 (5-6): 374-85.

27. Whitehead $M$. The concepts and principles of equity and health. Health Promotion International 1991; 6 (3): 217. 